\title{
Development of an allogeneic adherent stem cell therapy for treatment of ischemic stroke
}

\author{
Robert W. Mays, Ph.D. ${ }^{1 *}$; Cesar V. Borlongan Ph.D. ${ }^{2,3,4}$; Takao Yasuhara Ph.D. ${ }^{2}$; Koichi Hara ${ }^{2}$; Mina Maki ${ }^{2}$; \\ James E. Carroll M.D. ${ }^{2,4}$; Robert J. Deans Ph.D. ${ }^{1}$; David C. Hess M.D. ${ }^{2,4}$ \\ ${ }^{1}$ Regenerative Medicine, Athersys, Inc., Cleveland, OH 44115 USA \\ ${ }^{2}$ Department of Neurology, Med College of Georgia, Augusta, GA 30912 USA \\ ${ }^{3}$ Inst Molecular Medicine Genetics, Med College of Georgia, Augusta, GA 30912 USA \\ ${ }^{4}$ Research \& Affiliations Service Line, Augusta VAMC, GA 30912 USA
}

\begin{abstract}
To determine the potential for using stem cells in the treatment of ischemic injuries of the central nervous system, clinically relevant experiments were performed administering human adult adherent bone marrow derived stem cells into a rat model of ischemic stroke. Variables such as the requirement for immunosuppression, route of cell administration, window for therapeutic benefit and optimal cell dosage have all been examined in a series of relevant translational experiments in animals undergoing middle cerebral artery ligation stroke injury. Animals were tested for improvements in locomotor and neurological function at time points as late as 6 months post-cell transplantation and demonstrated sustained statistically significant benefit from a single dose of cells. Following sacrifice, immunohistochemistry was performed on tissues to determine stem cell engraftment and fate, as well as neuroprotection of endogenous tissue at the sites of ischemic injury. The results indicate locomotor and neurological improvement correlates with improved neuroprotection and limited retention of the transplanted stem cells, with limited neuronal fate. The observed benefit occurs in a cell dosage dependent fashion suggesting a pharmacological role for the cells when administered intravenously in these injury models.
\end{abstract}

Keywords: Stem and progenitor cells; ischemic stroke; translational research; xenogeneic graft; neuroprotection

\section{Introduction}

The treatment of stroke in adults represents a current unmet medical condition of significance in the United States and abroad. Stroke is the third leading cause of death and the leading cause for disability in the United States. There is currently only one therapy for treating stroke (recombinant tissue plasminogen activator), and even this approved therapy is limited by its narrow window of therapeutic efficacy. No cellbased therapeutics are currently on the market for treating stroke, which had an estimated cost of over $\$ 68.9$ billion dollars to patients in both direct and indirect costs in the United States alone in 2009 (LloydJones et al. 2009). Pre-clinical animal studies point to benefit derived from transplanted stem cells following ischemic injury in the CNS (Chen et al. 2001c; Chen et al. 2003b; Li and Chopp 2009; Ohtaki et al. 2008; Shyu et al. 2006; Yasuhara et al. 2008; Yasuhara et al. 2006b; Zhao et al. 2002), in the heart (reviewed in (Charwat et al. 2008; Ting et al. 2008)), the peripheral vasculature (Aranguren et al. 2008) and other inflammatory injury models (Aggarwal and Pittenger
2005; Kovacsovics-Bankowski et al. 2008; Kovacsovics-Bankowski et al. 2009).

It was recently demonstrated that the intravenous (IV) administration of multipotent adult progenitor cells (MAPC) provided equivalent recovery as cells injected directly into the brain of animals having undergone surgically induced neonatal hypoxic-ischemic (HI) injury, as measured by behavioral improvements and reduction in ischemic cell loss (Yasuhara et al. 2008). This finding supports the hypothesis for using MAPC, a unique allogeneic adult stem type, in treatment of individuals suffering an ischemic brain injury. In an effort to bring this therapy to the clinic, a set of clinically relevant optimization experiments using MultiStem ${ }^{\circledR}$, a proprietary human adult stem cell product isolated, grown and expanded under MAPC conditions, was performed to treat rats that had undergone surgically induced ischemic stroke injuries. The purpose of this study was to compare the behavioral benefits of these cells in the presence or the absence of immunosuppressive agents, to determine the optimal route of administration of the cells, to un-

\section{* Correspondence should be sent to:}

Dr. Robert W. Mays, Ph.D., Department of Regenerative Medicine, Athersys, Inc., 3201 Carnegie Avenue, Cleveland, OH 44115-2634. Tel: 216-431-9900 x 243; FAX: 216-361-9596; Email: rwmays@athersys.com 
derstand the time course of benefit for cell administration and to determine the optimal dose level for demonstrating efficacy for recovery. All experiments performed in the study were focused on effectively translating the observed therapeutic benefit of these cells in animal studies towards a clinical setting for treatment of patients suffering an ischemic stroke.

\section{Methods}

\subsection{Animals and ischemic stroke surgical procedures}

All animals were handled under Medical College of Georgia (MCG) ACORP protocol \#0205-001, and animal sacrifice, tissue harvest, fixation and immunohistochemistry performed at MCG. Adult, male Sprague Dawley rats were used in all procedures. Prior to stroke surgery, none of the animals differed in their motor and neurologic performance (baseline). After stroke, animals that reached the pre-set criterion of $>75 \%$ biased swing activity in elevated body swing test (EBST) and $>2.5$ neurologic deficit score in Bederson test were included for subsequent transplant experiments (post-stroke).

All surgical procedures were conducted under aseptic conditions. The animals were anesthetized with equithesin (300 mg/kg, i.p.) and checked for pain reflexes. Under deep anesthesia, animals underwent the Middle Cerebral Artery (MCA) ligation surgery. The MCA ligation technique involved identifying and isolating the bilateral common carotid arteries (CCA) through a ventral midline cervical incision. The CCA were ligated with non-traumatic arterial clips. A craniotomy of $-2 \times 2 \mathrm{~mm}^{2}$ was made in the right squamosal bone. The right MCA distal to the lenticulostriate branches was ligated for one hour with a 10-0 suture; thereafter the suture was removed and reperfusion ensued. The craniotomy was then covered with gelfoam. A heating pad and a rectal thermometer allowed maintenance of body temperature at normal limits. Laser Doppler measurements were used to verify arterial ligation, with the Doppler probe placed at the distal end of the MCA to measure cerebral blood flow before, during and after occlusion. A decrease of $>80 \%$ of blood flow compared to baseline was used to describe successful occlusion, with successful reperfusion described as $>50 \%$ of baseline. Only animals who successfully achieved $>80 \%$ occlusion and $>50 \%$ reperfusion were utilized in the study. This surgical model has been used successfully in previously published rat stroke studies (Chiang et al. 1999; Wang et al. 2001).

\subsection{Cell preparation}

MultiStem used in this study were banked and validated at Athersys, Inc. Cells (rat and human) were shipped to MCG on dry ice and stored in liquid nitrogen until reconstituted for the experiments. Frozen vials containing the required numbers of MultiStem were removed from the liquid nitrogen freezer. The vial information was recorded and vials were thawed in a $37^{\circ} \mathrm{C}$ water bath. Once the ice crystals disappeared from the vials, they were removed from the water bath and placed into a biological safety cabinet. Each vial was rinsed with $70 \%$ ethanol and wiped with a sterile kimwipe. The contents of the vial were transferred to a $15 \mathrm{~mL}$ conical centrifuge tube. $10 \mathrm{~mL}$ of pre-warmed media was added to the centrifuge tube that contained the cells. The tube was then centrifuged at $500 \mathrm{xg}$ for 5 minutes at room temperature. The supernatant was removed from the tube and the pellet re-suspended in media for cell counting. After cell counting, the tube was centrifuged at $500 \times \mathrm{xg}$ for 5 minutes at room temperature. The supernatant was aspirated and the cell pellet was re-suspended in PBS to cell concentrations meeting the dose requirements for the experiment.

\subsection{MultiStem transplantation (IC and IV)}

All surgical procedures were conducted under aseptic conditions. Under equithesin ( $3 \mathrm{ml} / \mathrm{kg}$ i.p.) anesthesia (animals checked for pain reflexes), the animals were implanted with MultiStem either directly into the striatum $(0.5 \mathrm{~mm}$ anterior to bregma, $2.8 \mathrm{~mm}$ lateral to midline and $5.0 \mathrm{~mm}$ below the dural surface) using a 28-gauge implantation cannula (Borlongan et al. 1998c; Borlongan et al. 1998d). Cells were thawed just prior to transplantation surgery as described in section 4.2 above. Viability cell counts, using Trypan Blue exclusion method, were conducted prior to transplantation and immediately after the transplantation on the last animal recipient to demonstrate cells were still $>80 \%$ viable. The cell dosages described refer to number of viable cells. Transplantation surgery was carried out within 2 hours after thawing the cells. The infusion rate of the cells into the animals was $1 \mu \mathrm{l}$ of cell solution per minute. Total volume infused was $3 \mu \mathrm{l}$ per animal. Following infusion, a 3minute absorption period was allowed before the needle was retracted. A heating pad and a rectal thermometer were used to maintain body temperature at $37^{\circ} \mathrm{C}$ throughout surgery and following recovery from anesthesia.

For IV infusion, following equithesin (3 $\mathrm{ml} / \mathrm{kg}$ i.p.) anesthesia (animals checked for pain reflexes), animals received transplantation directly into the exposed jugular vein in a total volume of $50 \mu$ of PBS over an infusion period of 10 minutes. A heating pad and a rectal thermometer were used to maintain body temperature at $37^{\circ} \mathrm{C}$ throughout surgery and following recovery from anesthesia. Transplantation was carried out within 2 hours after thawing the cells regardless of the route of administration.

\subsection{Locomotor and neurological testing}

-35 -

J Exp Stroke Transl Med (2010) 3(1): 34-46

Society for Experimental Stroke (www.s4es.org) 
All animal observers were blinded to the treatment of the animal at the time of evaluation. Animals were subjected to the elevated body swing test (EBST) and a 4-point neurological exam called the Bederson test. These tests have been shown to be sensitive assays of motor/sensory deficits produced by unilateral stroke surgery (Borlongan et al. 1998a; Borlongan and Sanberg 1995; Borlongan et al. 1998b; Borlongan et al. 1998d). The EBST involves handling the animal by its tail and recording the direction of the swings. The test apparatus consisted of a clear Plexiglas box $(40 \times 40 \times 35.5 \mathrm{~cm})$. The animal was gently picked up at the base of the tail, and elevated by the tail until the animal's nose is at a height of 2 inches (5 $\mathrm{cm}$ ) above the surface of the box. The direction of the swing, either left or right, was counted once the animals head moved sideways approximately 10 degrees from the midline position of the body. After a single swing, the animal was placed back in the Plexiglas box and allowed to move freely for 30 seconds prior to retesting. These steps are repeated 20 times for each animal. Normally, intact rats display a $50 \%$ swing bias, that is, the same number of swings to the left and to the right. A 75\% swing bias would indicate 15 swings in one direction and 5 in the other during 20 trials. Previously the EBST has been demonstrated to be an effective tool in demonstrating stable asymmetric deficits in lesioned animals for up to six months (Borlongan and Sanberg 1995).

One hour after the EBST, a neurological exam called the Bederson Test was conducted following procedures previously described (Altumbabic et al. 1998; Bederson et al. 1986). The neurological score for each rat was obtained using 4 tests which include (1) observation of spontaneous ipsilateral circling, graded from 0 (no circling) to 3 (continuous circling); (2) contralateral hindlimb retraction, which measures the ability of the animal to replace the hindlimb after it is displaced laterally by 2 to $3 \mathrm{~cm}$, graded from 0 (immediate replacement) to 3 (replacement after minutes or no replacement); (3) beam walking ability, graded 0 for a rat that readily traverses a 2.4-cm-wide, 80$\mathrm{cm}$-long beam to 3 for a rat unable to stay on the beam for 10 seconds; and (4) bilateral forepaw grasp, which measured the ability to hold onto a 2-mmdiameter steel rod, graded 0 for a rat with normal forepaw grasping behavior to 3 for a rat unable to grasp with the forepaws. The scores from all 4 tests, which were performed over a period of about 15 minutes on each assessment day, were added to give a neurological deficit score (maximum possible score, 12) and then divided by 4 (the number of individual tasks) to give an average neurological deficit for each animal.

\subsection{Immunohistochemical and histological analyses}

At the conclusion of in life testing, animals were sacrificed for histology and immunohistochemistry. Some slices were processed using hematoxylin and eosin (H\&E) and Nissl staining of brain sections. Alternate slices of tissues were processed using standard $A B C$ methods using modified procedures. Briefly, $20 \mu \mathrm{m}$ cryostat sectioned tissues were examined at $4 X$ magnification and digitized using a PC-based Image Tools computer program. Brain sections were blindcoded and Abercrombie's formula was used to calculate the total number of immuno-positive cells. This formula allows for a downward correction of stained cells normally associated with observer bias. The Borlongan laboratory routinely performed such quantification of quantifying transplanted cells (Borlongan et al. 2000; Borlongan et al. 2001).

\subsection{Statistical analyses}

Using power calculations [(1-Beta) 0.80] with alpha set at 0.05 , the number of animals indicated generated the minimum number of data sets necessary to run statistical analyses. The behavioral scores (EBST and Bederson Test) and graft survival (Abercrombie corrected) were analyzed with One-way ANOVA. Pairwise comparisons were made between treatment groups by Protected Fisher's LSD. The level of significance was set at $p<0.05$.

\subsection{Experimental designs}

\subsubsection{Immunosuppression experiment}

Thirty-five Sprague Dawley (SD) rats underwent middle cerebral artery (MCA) ligation surgery to induce a surgical stroke in the animals. Seven days after the induction of stroke, animals were randomized into five cohorts of seven animals each, with each cohort receiving direct intra-cerebral administration of one of the following treatments: 1) $3 \mu \mathrm{l}$ injection of PBS containing 400,000 allogeneic rat MAPC with no immunosuppression, 2) $3 \mu \mathrm{l}$ injection of PBS containing 400,000 allogeneic rat MAPC with immunosuppressive treatment (CsA, $1 \mathrm{mg} / \mathrm{kg}$, intraperotineal), 3) $3 \mu \mathrm{l}$ injection of PBS containing 400,000 human xenogeneic MultiStem with no immunosuppression, 4) $3 \mu \mathrm{l}$ injection of PBS containing 400,000 xenogeneic human MultiStem with immunosuppressive treatment (CsA, $1 \mathrm{mg} / \mathrm{kg}$, i.p.), 5) $3 \mu \mathrm{l}$ injection of PBS containing 400,000 irradiated, non-viable xenogeneic human MultiStem with immunosuppressive treatment (CsA, $1 \mathrm{mg} / \mathrm{kg}$, i.p.). At 14 days after cell transplantation, and every 14 days thereafter for 8 weeks, each animal underwent an EBST and Bederson Test to determine locomotor and neurological function.

\subsubsection{Route of administration experiment}

Forty-two SD rats underwent middle cerebral artery (MCA) ligation surgery to induce a surgical stroke in the animals. Seven days after the induction of stroke, -36 - 
animals were randomized into six cohorts of seven animals each, with each cohort receiving intravenous administration of one of the following treatments: 1) 400,000 human MultiStem with immunosuppressive treatment (CsA, 1mg/kg, intraperotineal), 2) 400,000 human MultiStem with no immunosuppression, 3) 1 Million human MultiStem with immunosuppressive treatment (CsA, $1 \mathrm{mg} / \mathrm{kg}$, i.p.) 4) 1 Million human MultiStem with no immunosuppressive treatment 5) 1 Million irradiated, non-viable human MultiStem with immunosuppressive treatment (CsA, $1 \mathrm{mg} / \mathrm{kg}$, intraperotineal), or 6) 1 Million irradiated, non-viable human MultiStem with no immunosuppressive treatment. At 14 days after cell transplantation, and every 14 days thereafter for 8 weeks, each animal underwent an EBST and Bederson Test to determine locomotor and neurological function.

\subsubsection{Window of therapeutic benefit experiment}

Twenty-Eight SD rats underwent middle cerebral artery MCA ligation surgery to induce a surgical stroke in the animals. The animals were randomized into four groups of seven animals each, with each group receiving intravenous administration of 1 Million Xenogeneic MultiStem. No immunosuppression was used on any animals in this experiment. Group 1 animals received cells 1 day after induction of ischemic stroke, Group 2 animals received cells 2 days after induction of ischemic stroke, Group 3 animals received cells on Day 7 after induction of ischemic stroke, and Group 4 animals received 1 Million nonviable irradiated cells on Day 7 after induction of ischemic stroke. At 14 days post cell transplantation, and every 14 days thereafter for 8 weeks, each animal underwent an EBST and Bederson Test to determine locomotor and neurological function. Animals were sacrificed after testing on day 56 posttransplantation and brains were harvested, fixed and then stained for analysis by immunohistochemistry and microscopic analyis to determine MultiStem engraftment or Nissl staining to determine endogenous neuronal protection.

\subsubsection{Dose escalation experiment}

Forty-nine Sprague-Dawley rats underwent a surgical ligation of the distal middle cerebral artery to induce an ischemic stroke in the cortex of the animal. 2 days post-injury animals were randomly assigned to one of 7 groups. All cells were transplanted via intravenous administration into the jugular vein. No animals received immunosuppression at any point in the study. Rats received either 1 million irradiated non-viable MultiStem as a negative control, 400,000, 1 million, 2 million, 4 million, 10 million or 20 million xenogeneic human MultiStem. All of the animals were evaluated for locomotor performance and neurological deficits using the EBST and the composite Bederson test, respectively on day 14 post-cell transplantation and then every other 14 days afterwards throughout the "in life" portion of the study. Following testing on day 84 post-transplantation, animals were sacrificed and evaluations of transplanted cell persistence, and endogenous neuroprotection were performed using immunohistochemistry and microscopy techniques.

\section{Results}

\subsection{Requirement for immunosuppression}

As shown in Figure $\mathbf{1}$ and Figure 2, regardless of whether xenogeneic or allogeneic cells are transplanted or whether CsA is used, animals receiving administration of the stem cells show sustained and statistically significant improvements in both the tests. These data demonstrate that immunosuppression is not required in conjunction with MultiStem as a therapeutic, and that cells transplanted 7 days after ischemic stroke injury provide sustained (8-week) and statistically significant benefit.

\subsection{Route of administration}

As shown in Figure 3, locomotor benefit is dose dependent as only animals infused IV with 1 Million cells show any sustained improvement versus nonviable control cells. However, significant neurological benefit is observed at doses of both 400,000 and 1 Million transplanted cells, although there is a trend of animals treated with 400,000 cells to show a decline in recovery over the 56 day time period. Animals treated at a dose of 1 Million cells show sustained and statistically significant improvement in both tests over the entire 8 week time course of the study. Although only the animals receiving cells in the absence of immunosuppression are illustrated in Figure 3 , there was no difference between animals that did or did not receive CsA in either the EBST or Bederson tests (data not shown).

\subsection{Window of therapeutic benefit}

Evaluation of locomotor and neurological skills in rats receiving 1 Million xenogeneic MultiStem Intravenous at different times after induction of ischemic stroke. There is a sustained and statistically significant improvement in all groups receiving 1 Million viable MultiStem regardless of when the cells are delivered as demonstrated in both locomotor and neurological tests, compared to the non-viable control (Figure 4). There was no statistical difference between the different experimental groups when comparing the EBST and Bederson test results. These data demonstrate that IV administration of MultiStem can provide benefit in ischemic brain injuries when administered in a window from 1 to 7 days following the injury. 


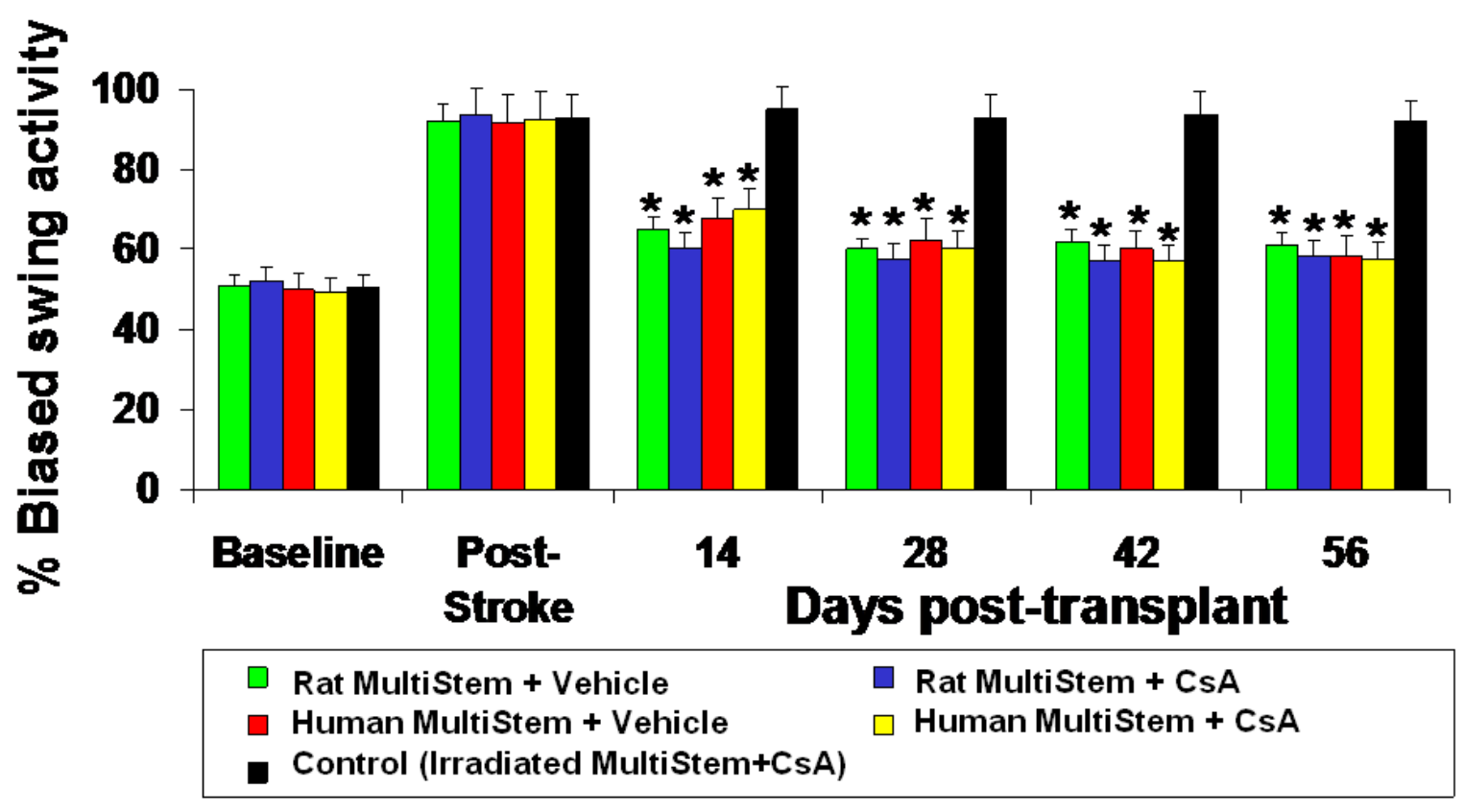

Figure 1: Xenogeneic and allogeneic MultiStem promote sustained and statistically significant locomotor recovery following ischemic stroke in rats. Rats underwent a distal middle cerebral artery ligation surgery to induce a focal ischemic stroke. 7 days after induction of the stroke injury, rats were randomly placed into one of 5 treatment groups. Eight animals per group received either 400,000 allogeneic rat MultiStem via intracranial injection with or without Cyclosporine A treatment (CsA) or 400,000 xenogeneic human MultiStem cells with or without Cyclosporine A treatment. 400,000 irradiated non-viable human MultiStem were transplanted into the negative control animals. The Elevated Body Swing Test (EBST) was performed to demonstrate locomotor outcomes every 14 days post-transplantation for 8 weeks. The asterisks indicate a significant difference between the negative control treatment group and the MultiStem experimental groups (One-Way ANOVA, $p<0.05$; Fisher's PLSD, $p<0.05$ ).

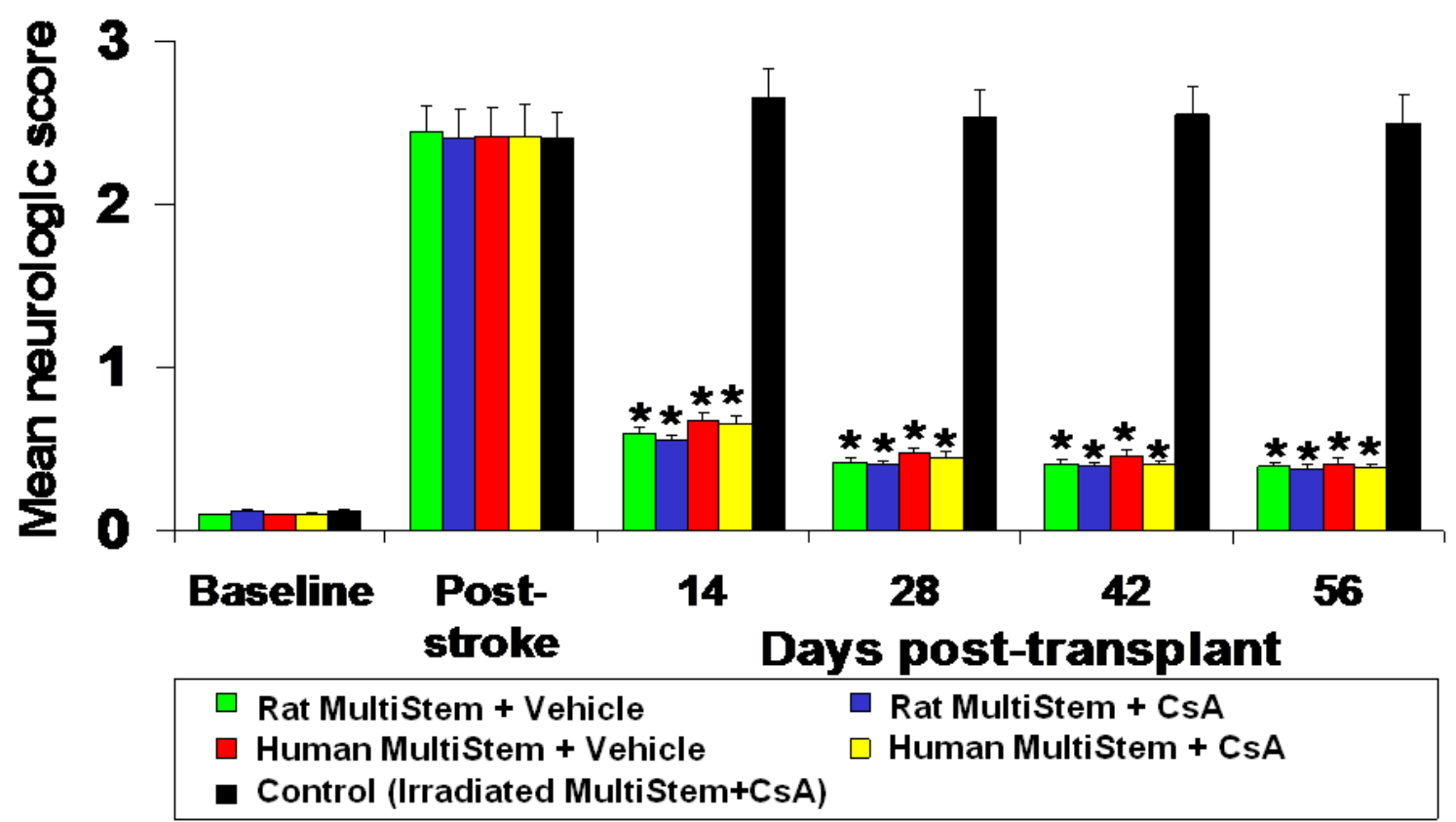

Figure 2: Transplanted allogeneic and xenogeneic MultiStem cells provide sustained and significant neurological benefit in rat model of stroke without requirement for immunosuppression._Rats un- 38 - 
derwent a distal middle cerebral artery ligation surgery to induce a focal ischemic stroke. 7 days after induction of the stroke injury, rats were randomly placed into one of 5 treatment groups. Eight animals per group received either 400,000 allogeneic rat MultiStem via intracranial injection with or without Cyclosporine $A$ treatment (CsA) or 400,000 xenogeneic human MultiStem cells with or without Cyclosporine A treatment. 400,000 irradiated non-viable human MultiStem were transplanted into the negative control animals. Behavioral tests (Bederson composite tests) for neurological functions were conducted every 14 days posttransplantation for 8 weeks. The asterisks indicate a significant difference between the negative control treatment group and the MultiStem experimental groups (One-Way ANOVA, $p<0.05$; Fisher's PLSD, $p<$ 0.05).

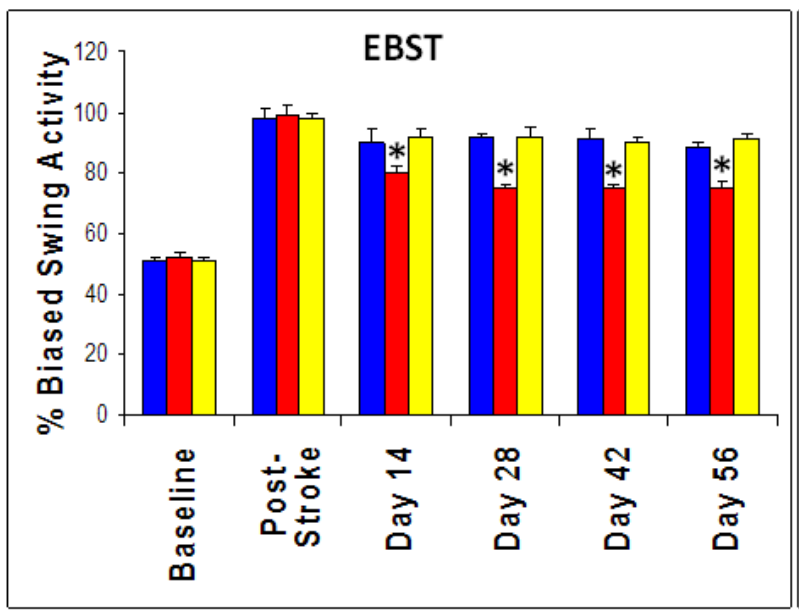

400,000 MultiStem

\section{Million MultiStem}

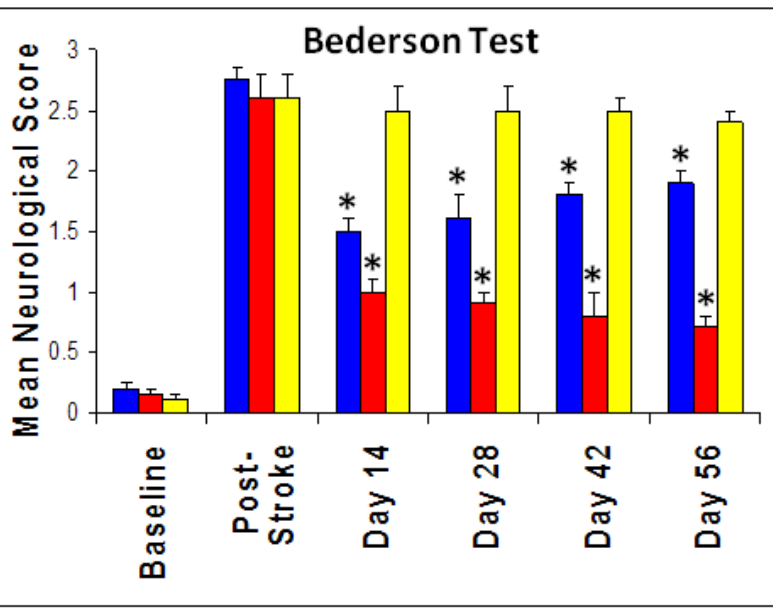

1 Million Non-Viable MultiStem

Figure 3: IV transplanted xenogeneic MultiStem cells provide motor and neurological benefit in rat mcal model of stroke. Rats which had undergone the MCAL surgically induced stroke, received 400K MultiStem 7-days post injury (blue bars), 1 Million MultiStem 7-days post injury (red bars) or 1 Million irradiated non-viable MultiStem 7 days post-injury (yellow bars) via direct IV infusion into the jugular vein. Animals were then subjected to EBST measurements or Bederson testing every 14 days after cell transplantation for 8 weeks. The asterisk indicates where animals that received MultiStem showed a significant $(p<0.05)$ improvement over the negative control treated animal group (One-Way ANOVA, $p<0.05$; Fisher's PLSD, $p<$ $0.05)$. All data in this figure were from animals which received no immunosuppression.

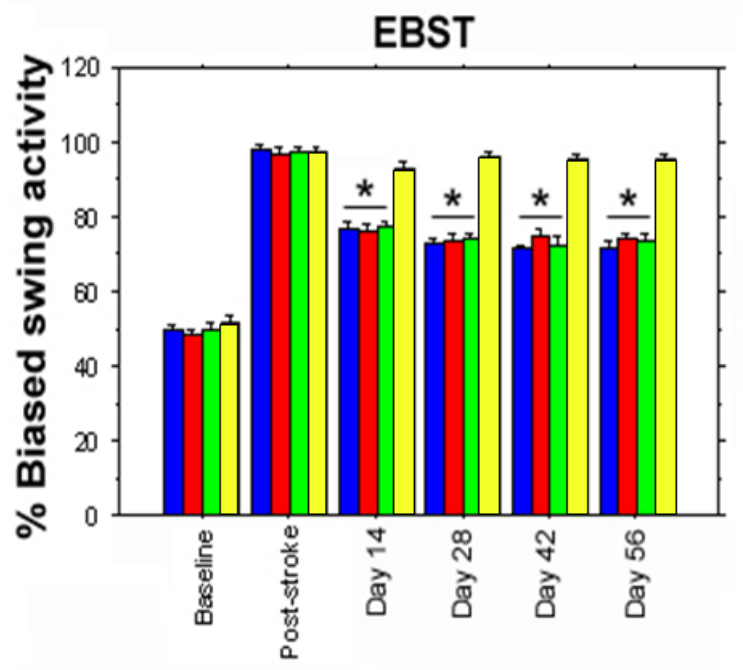

Cell Delivery on Day 1

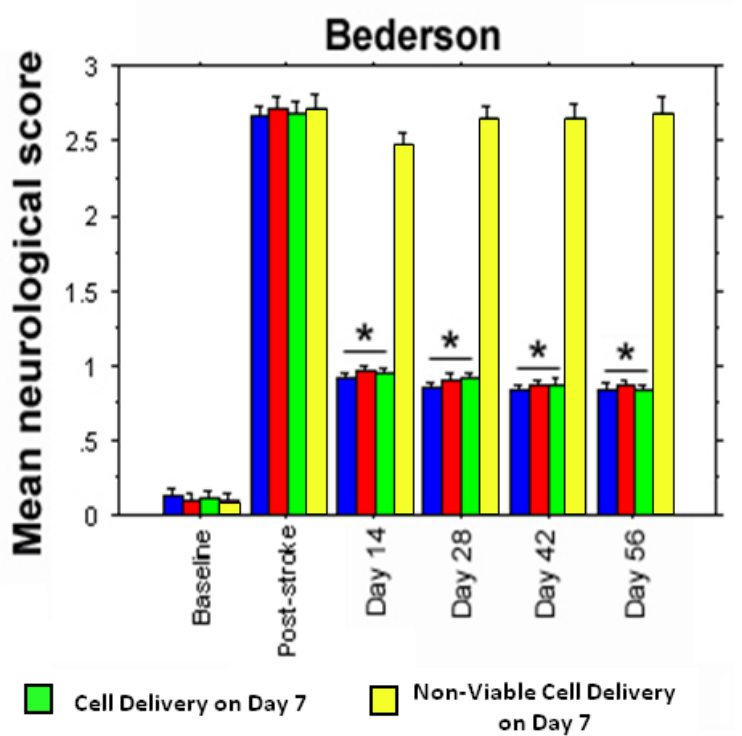


Figure 4: Transplanted xenogeneic MultiStem cells provide sustained and statistically significant motor and neurological benefit in rat mcal model of stroke when delivered 1, 2 or 7 days post-injury. Rats which had undergone surgical MCAL induced stroke, received 1 million MultiStem intravenously 1-day post-injury (blue bars), 2-days post-injury (red bars), 7-days post-injury (green bars) or 1 million irradiated non-viable MultiStem cells 7 days post-injury (pink bars). Animals were then subjected to EBST measurements or Bederson testing every 14 days after cell transplantation. The asterisks indicate that the animal groups that received xenogeneic MultiStem (Days 1,2 and 7 ) all showed a significant $(p<0.05)$ improvement over the control group (One-Way ANOVA, $p<0.05$; Fisher's PLSD, $p<0.05$ ). There was no statistical difference between cell treated animal groups at any time point

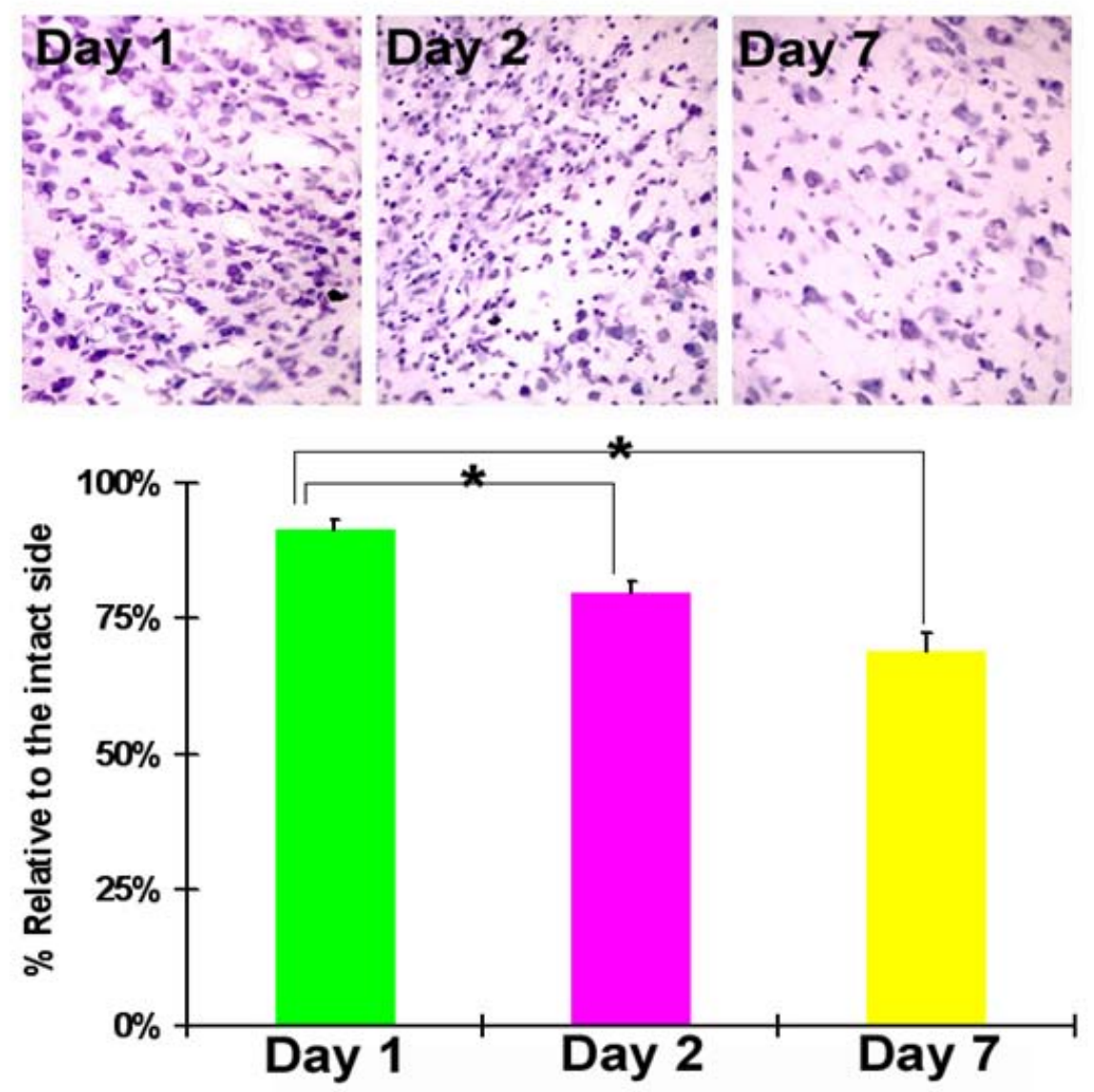

Figure 5: Temporal reduction in endogenous neuronal cell loss following iv infusion of MultiStem into rats suffering ischemic stroke. Following behavioral testing on Day 56 post-MultiStem infusion, animals that had undergone a surgical MCAL induced ischemic stroke were sacrificed and brain section were Nissl stained to compare endogenous neuronal viability of engrafted animals receiving cells at different times after stroke. Viable cells per field were counted and compared to the counts from the uninjured contralateral field on the same section (Uninjured contralateral cell counts set to 100\%). Data demonstrates statistically significant protection of neurons in the penumbral region following MultiStem transplantation. Asterisks indicate statistical significance at $p<0.05$ versus other groups (One-Way ANOVA, $p<0.05$; Fisher's PLSD, $p<0.05$ ). 


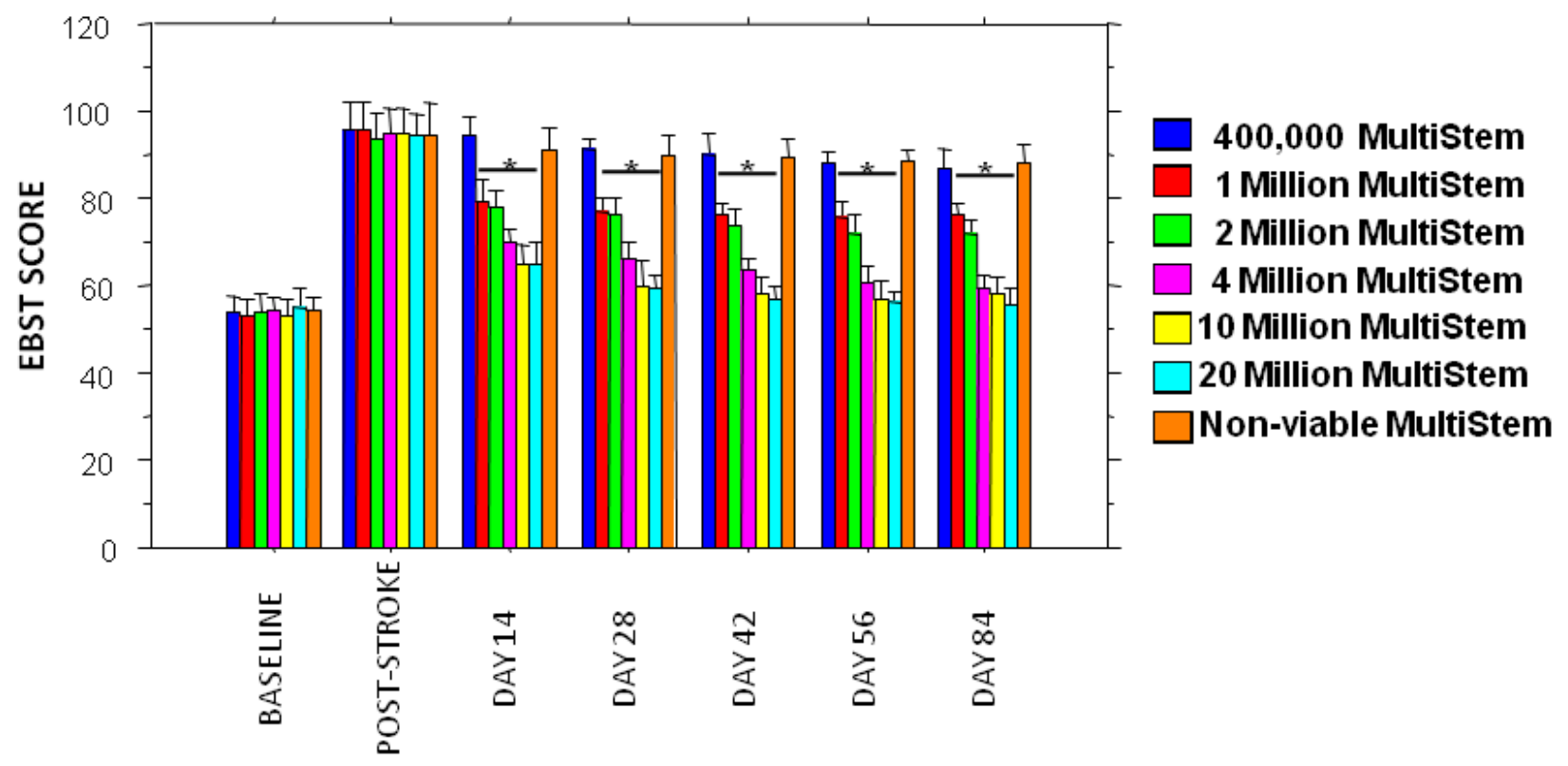

Figure 6. Dose dependent improvement in locomotor testing following IV administration of human MultiStem in rat mcal model of stroke. Rats which had undergone MCAL surgically induced stroke, received the indicated dose of human MultiStem 2-days post-injury. Animals were then subjected to EBST measurements every 14 days after cell transplantation for 12 weeks. The asterisks indicate the animal groups that showed a significant $(p<0.05)$ improvement over the control group (One-Way ANOVA, $p<0.05$; Fisher's PLSD, $\mathrm{p}<0.05$ ). Behavioral tests for locomotor function were conducted prior to surgery (BASELINE), after stroke surgery and recovery but prior to cell administration (POST-STROKE) for reference.

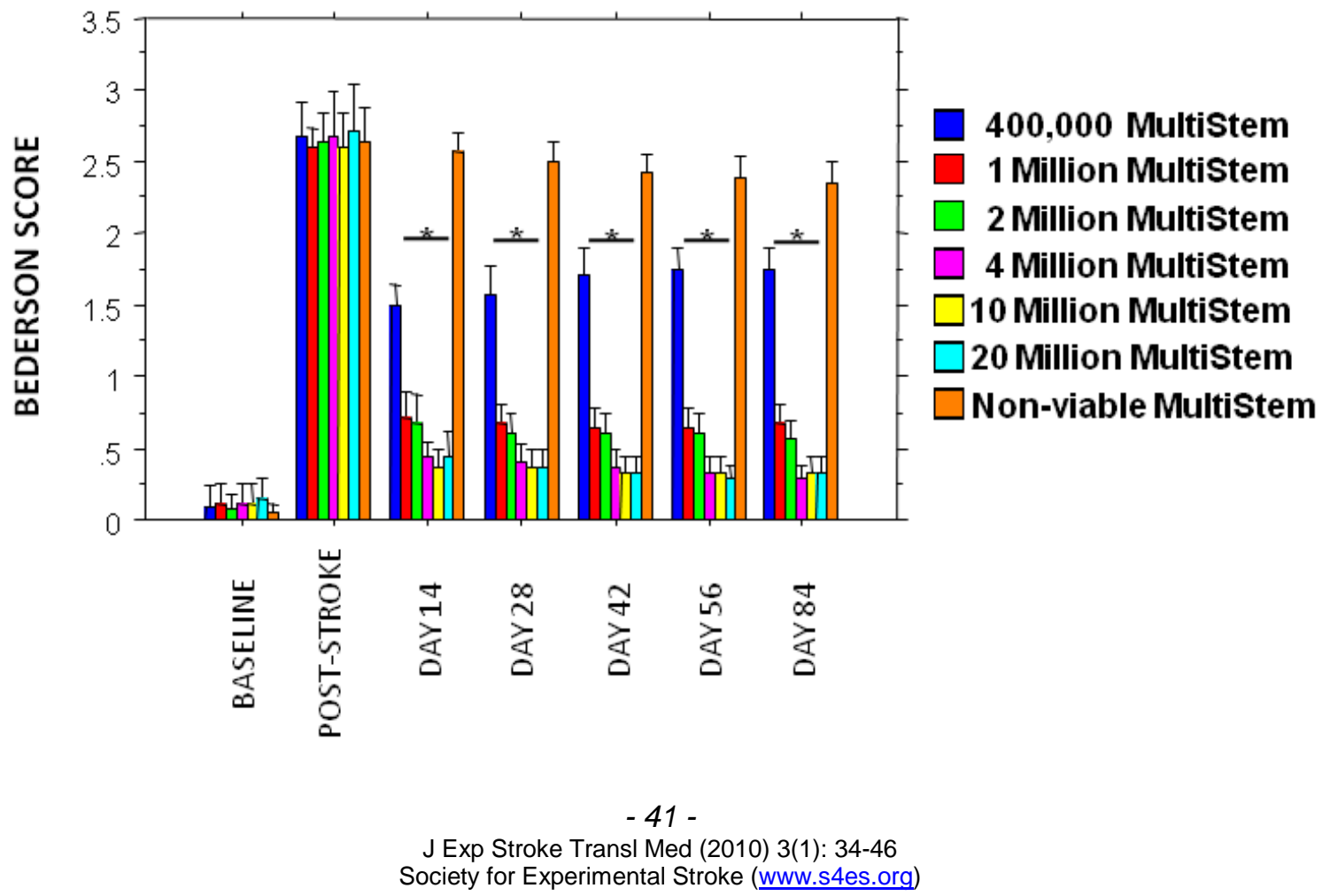


Figure 7. Dose dependent improvement in neurological testing in rats following IV administration of MultiStem in rat mcal model of stroke. Rats which had undergone MCAL surgically induced stroke, received the indicated dose of human MultiStem 2-days post-injury. Animals were then subjected to Bederson Composite Testing every 14 days after cell transplantation for 12 weeks. All dosage groups at all time points showed a statistically significant $(p<0.05)$ improvement over the control group (One-Way ANOVA, $p<$ 0.05; Fisher's PLSD, $p<0.05$ ). Testing for neurological functio were conducted prior to surgery (BASELINE), after stroke surgery and recovery but prior to cell administration (POST-STROKE) for reference.

Histological analysis of MultiStem engraftment in ischemic stroke rat brain following IV Infusion at 1, 2 and 7 days post-injury. Following the last behavioral testing at day 56 for each group, animals were sacrificed and the brains were harvested. Semi-thin sections of paraffin embedded tissue were stained with DAPI to visualize all cell nuclei and mouse anti-HuNu (human nuclei) polyclonal antibodies, followed by FITC-conjugated goat anti-mouse monoclonal antibodies to stain engrafted human MultiStem. The total number of engrafted cells counted was then calculated as a percentage of total number of cells infused per animal (data not shown). Although not statistically significant there is a trend showing that the earlier the cells were infused after injury, the fewer viable engrafted cells remain. One day after injury had an average of $0.75 \%$ engraftment, 2 days after injury had an average of $1.1 \%$ engrafted cells while infusion 7 days after injury had $1.27 \%$ viable engrafted cells. These data suggest that the hostile inflammatory environment present near the ischemic injury after stroke may be less favorable for engraftment and long term survival of the infused MultiStem.

Earlier Infusion of MultiStem promotes ilncreased endogenous neuronal protection following stroke. In addition to determining the level of graft survival present in the ischemic brain at day 56 post cell infusion, alternate sections were Nissl stained to determine the level of endogenous neuronal viability. There was a statistically significant decrease in endogenous neuronal death the earlier the MultiStem were infused (Figure 5). There was a statistical difference between viable neurons in animals receiving MultiStem on Day 1 vs. Day 2 after stroke, as well as statistical significance between animals receiving cells on Day 1 vs. Day 7 . These results suggest that the earlier cells are delivered following an ischemic event, the more benefit the cells may be able to exert in protecting endogenous neurons and the peri-infarct region.

\subsection{Dose escalation}

Evaluation of locomotor and neurological skills in rats receiving different doses of xenogeneic MultiStem after induction of ischemic stroke. Transplantation with human MultiStem resulted in a significant $(p<0.05)$ improvement over control for EBST locomotion and Bederson neurological function. It is clear that there is a dose response effect of MultiStem, with increasing effectiveness at higher doses. This effect began 14 days post-transplant and was maintained through Day 84. The magnitude of the improvement was rapid as evidenced in the lower doses increasing from $400 \mathrm{k}$ to about 4 million cells, subsequently the rate of improvement slowed and tended to plateau for the 10 and 20 million cell doses. These results are presented graphically for the EBST (Figure 6) and Bederson composite (Figure 7).

\section{Discussion}

Over the past decade, a number of different adult stem cell types have been isolated and subsequently tested in pre-clinical models of ischemic brain injury or stroke with encouraging results (Chen et al. 2001a; Chen et al. 2003b; Li and Chopp 2009; Ohtaki et al. 2008; Shyu et al. 2006; Yasuhara et al. 2006b; Zhao et al. 2002). Much of the early pre-clinical work performed by Chopp and colleagues used marrow derived stromal cells. Reports demonstrated that transplantation of marrow derived stromal cells improve functional outcome in a dose-response fashion in rodent middle cerebral artery occlusion models when given intracerebrally, intra-arterially, or intravenously (Chen et al. 2001a; Chen et al. 2001b; Li et al. 2001). Intravenous transplantation is effective at improving functional outcome even when given as late as one month after the insult (Shen et al. 2007). There was no reduction of infarct size; instead a neurorestorative effect was observed with increases in angiogenesis, neurogenesis and synaptogenesis in the marrow stromal cell-treated groups (Chen et al. 2003a; Chen et al. 2003b).

MultiStem $\AA$, a proprietary stem cell product, represents a unique population of adherent bone marrow derived cells and testing and validation in pre-clinical models of injury and disease must be confirmed independently from other bone marrow derived stem and stromal cells from which MultiStem has separate and distinct biology (Boozer et al. 2009). MultiStem is being developed for the treatment of multiple disease indications, and the Food and Drug Administration has already allowed use of this cell product in Phase I clinical trials including treatment of acute myocardial infarct and prophylaxis against graft -42 -

J Exp Stroke Transl Med (2010) 3(1): 34-46

Society for Experimental Stroke (www.s4es.org) 
vs. host disease, and these trials are currently enrolling patients. It has previously been demonstrated that these cells are effective in a neonatal rat model of hypoxic-ischemic brain injury (Yasuhara et al. 2008; Yasuhara et al. 2006a; Yasuhara et al. 2006b). In an effort to determine whether MultiStem is effective in treatment of ischemic stroke, a series of translationally relevant experiments were undertaken paralleling previous work performed with marrow derived stromal cells.

The results indicate that immunosuppression is not required for MultiStem to provide benefit in a rat model of ischemic stroke when delivered intracranially (Figures 1 and 2). The benefit is sustained and significant through the entire in life portion of the experiments, as measured by the EBST and composite Bederson testing. MultiStem has previously been shown to have minimal immunogenicity, allowing it to be used "off-the-shelf" without tissue matching or the need for immunosuppresion in providing benefit in a graft vs. host injury model (Kovacsovics-Bankowski et al. 2008), and the results of the current study support this biological feature of these cells.

In order to find a more a "clinically practical" route of administration compared to our initial intra-cranial delivery experiments, the efficacy of intravenous delivery of MultiStem over a limited dose range was compared in the rat surgical stroke model. Validation of such a minimally invasive delivery route would obviate the need for direct transplantation of the cells into the brain, thereby eliminating the need for a neurosurgical component in the clinic. Since therapeutic benefits were observed with an intracranial dose of 400,000 delivered cells, this dose was used as reference for our comparative IV studies. Intravenous infusion of 400,000 MultiStem produced partial benefits detected only in Bederson test, whereas the 1 million cell dose promoted more robust and stable behavioral recovery than controls in both tests (Figure 3 ). The benefits demonstrated by the 400,000 cell dose in the Bederson test, although significant at all time points tested, showed a trend towards loss of a sustained benefit as a function of time over the course of testing. This partial study also showed that immunosuppression is not required to produce benefits in an intravenous delivery paradigm as well. These findings support previously published reports demonstrating the efficacy in outcomes following intravenous administration of marrow stromal cells (Chen et al. 2001b) or umbilical cord blood stem cells (Chen et al. 2001c) in rat models of stroke. It has also been reported that intravenous administration of cord blood was more effective than intra-striatal administration in producing functional benefit following stroke in rats (Willing et al. 2003).
In view of the fact that 400,000 intravenously administered MultiStem resulted in producing only partial benefits, which appear to wane over time, when compared to 1 million cells, attempts were made to resolve this unstable functional recovery in two ways: by performing a more extensive dose ranging for the intravenously administered stem cells, as well as by understanding the time course of functional benefit for administering cells post-stroke. The theoretical rationale for early transplantation is based on the rapid deterioration of the brain after stroke, suggesting that immediate intervention is likely to provide relief and halt the disease progression. Subsequently, a new cohort of adult rats underwent the same stroketransplant paradigm, with modification of the timing of intravenous MultiStem treatment by transplanting animals at day 1,2 or 7 post-stroke or with irradiated non-viable MultiStem as a negative control at day 7 post-stroke. A one million cell dose was delivered intravenously in order to test the hypothesis that if early timing was effective, then it should enhance the functional effects comparable to higher doses of cells, as well as the fact that this dosage had demonstrated a sustained statistically significant benefit in the previous study. Regardless of timing, intravenous administration at days 1,2 or 7 poststroke resulted in a similar degree of behavioral recovery compared to controls, which was sustained up to 2 months post-transplant (Figure 4). However, histological examination revealed that day 1 delivery led to better protection of at risk cells in the periinfarct region ( $>80 \%$ of intact) than day 2 or $7(75 \%$ and $60 \%$ of intact; p's < 0.05; Figure 5). As alluded to in section 3.3 above, histological staining of alternate sections for the presence of engrafted MultiStem illustrates an inverse relationship between viable rat neurons and engrafted MultiStem. The earlier the MultiStem cells are administered, fewer MultiStem cells are detectable at Day 56 post-infusion, however the greater the level of host neuronal protection. Furthermore, preliminary unpublished shorter term (i.e. 1-7 day) studies suggest that the majority of MultiStem are cleared from the injury site within 48-72 hours. These data suggest that the therapeutic benefit MultiStem provides in this rodent model of stroke is unlikely to be from direct neuroreplacement. The potential mechanisms that may explain the observed neuroprotection are being actively pursued, with hypotheses that multiple trophic factors and downstream signaling pathways may be implicated.

One possible pathway through which MutliStem may provide survival benefit is through the Akt pathway. Although, the direct role of Akt pathway involvement by MultiStem following stroke remains to be demonstrated, it is interesting to note that both VEGF and BDNF, growth factors demonstrated to be expressed by MultiStem at the transcriptional (Hamilton, unpub-43 - 
lished results) and protein levels (van't Hof, unpublished results) in vitro, have been cited to activate Akt and provide neuroprotection following ischemic injury in vivo (Han and Holtzman 2000; Kilic et al. 2006). Similar trophic factor cascades and neuroprotective mechanisms have been proposed for the benefits conferred by infused marrow stromal cells in previously published rodent stroke studies (Chopp and Li 2002).

To more thoroughly understand the dose response following intravenous MultiStem administration in a stroke injury model, an extensive dose ranging experiment was performed in groups of stroke injured rats. Data from both locomotor and neurological tests illustrate that there is a dose dependent improvement in outcomes up to the 4 million cell dosage, at which point no further significant improvement is exhibited despite increases in intravenous administered cells up to 20 million total infused cells (Figures $\mathbf{6}$ and $\mathbf{7}$ ). The data supports the hypothesis that intravenous administered MultiStem provide a "pharmacologic"like effect that plateaus at higher cell dosages suggesting that at least some aspect of the benefit that the cells provide can be saturated above which no additional cells effect additional biological benefit.

It is proposed that MultiStem must be producing proteins or trophic modulators that are involved in one or more of the following biological processes: (1) overriding the basic processes of neuronal cell decline and death caused by the ischemic injury (limited oxygen, nutrients), (2) intervening in the neuroinflammatory process, (3) stimulating neoangiogenesis and improving circulation to the ischemic tissue, and/or (4) contributing indirectly to endogenous tissue regeneration, i.e. causing the recruitment or production of new endogenous cells to replace damaged cells. It is important therefore to identify and evaluate candidates produced by Multistem or affected by the presence of MultiStem which may participate in these processes using techniques such as proteomic and genomic analysis.

Cell therapy for stroke represents an important paradigm in treatment of acute ischemic injury, in the context of a multi-modality approach to ischemic injury (Stem Cell Therapies as an Emerging Paradigm in Stroke (STEPS): bridging basic and clinical science for cellular and neurogenic factor therapy in treating stroke 2009). Given the failure of many single modality drugs, it is important to assess this new approach in the translational setting. The FDA recently granted approval to initiate a Phase I safety evaluation for MultiStem infusion at day 2 following ischemic stroke. That clinical study as well as the evaluation of bone marrow mononuclear cells (Savitz 2009) or gene modified neuronal stem cells (Stroemer et al. 2009) will comprise an important translational dataset to resolve this important unmet clinical need.

\section{Acknowledgement}

We would like to thank Anthony Ting, Gil van Bokkelen, Jason Hamilton, Jef Pinxteren and Nick Lehman for invaluable discussion, technical assistance, and review of this manuscript. Partial support for this work was provided by a grant from the State of Ohio Biomedical Research Commercialization Program, award \#06-063. This work was supported by a translational $\mathrm{U}$ grant from $\mathrm{NINDS} / \mathrm{NIH}$, grant \# 1 U01 NS055914-01A2.

\section{Conflict of interest}

RWM and RJD are both employed by Athersys, Inc. and have financial stake in the company.

\section{References}

Aggarwal S, Pittenger MF. (2005) Human mesenchymal stem cells modulate allogeneic immune cell responses. Blood 105:1815-1822

Altumbabic M, Peeling J, Del Bigio MR. (1998) Intracerebral hemorrhage in the rat: effects of hematoma aspiration. Stroke 29:1917-1922; discussion 1922-1913

Aranguren XL, McCue JD, Hendrickx B, Zhu XH, Du F, Chen E, Pelacho B, Penuelas I, Abizanda G, Uriz M, Frommer SA, Ross JJ, Schroeder BA, Seaborn MS, Adney JR, Hagenbrock J, Harris NH, Zhang Y, Zhang $X$, Nelson-Holte $M H$, Jiang $Y$, Billiau $A D$, Chen $W$, Prosper F, Verfaillie CM, Luttun A. (2008) Multipotent adult progenitor cells sustain function of ischemic limbs in mice. J Clin Invest 118:505-514

Bederson JB, Pitts LH, Tsuji M, Nishimura MC, Davis RL, Bartkowski H. (1986) Rat middle cerebral artery occlusion: evaluation of the model and development of a neurologic examination. Stroke 17:472-476

Boozer S, Lehman N, Lakshmipathy U, Love B, Raber A, Maitra A, Deans R, Rao MS, Ting AE. (2009) Global Characterization and Genomic Stability of Human MultiStem, A Multipotent Adult Progenitor Cell. Journal of Stem Cells 4:17-28

Borlongan CV, Hida H, Nishino H. (1998a) Early assessment of motor dysfunctions aids in successful occlusion of the middle cerebral artery. Neuroreport 9:36153621

Borlongan CV, Sanberg PR. (1995) Elevated body swing test: a new behavioral parameter for rats with 6hydroxydopamine-induced hemiparkinsonism. J Neurosci 15:5372-5378

Borlongan CV, Saporta S, Poulos SG, Othberg A, Sanberg PR. (1998b) Viability and survival of hNT neurons determine degree of functional recovery in grafted ischemic rats. Neuroreport 9:2837-2842

Borlongan CV, Stahl CE, Keep MF, Elmer E, Watanabe S. (2000) Cyclosporine-A enhances choline acetyltransferase immunoreactivity in the septal region of adult rats. Neurosci Lett 279:73-76 
Borlongan CV, Su TP, Wang Y. (2001) Delta opioid peptide augments functional effects and intrastriatal graft survival of rat fetal ventral mesencephalic cells. Cell Transplant 10:53-58

Borlongan CV, Tajima Y, Trojanowski JQ, Lee VM, Sanberg PR. (1998c) Cerebral ischemia and CNS transplantation: differential effects of grafted fetal rat striatal cells and human neurons derived from a clonal cell line. Neuroreport 9:3703-3709

Borlongan CV, Tajima Y, Trojanowski JQ, Lee VM, Sanberg PR. (1998d) Transplantation of cryopreserved human embryonal carcinoma-derived neurons (NT2N cells) promotes functional recovery in ischemic rats. Exp Neurol 149:310-321

Charwat S, Gyongyosi M, Lang I, Graf S, Beran G, Hemetsberger R, Nyolczas N, Sochor H, Glogar D. (2008) Role of adult bone marrow stem cells in the repair of ischemic myocardium: current state of the art. Exp Hematol 36:672-680

Chen J, Li Y, Katakowski M, Chen X, Wang L, Lu D, Lu M, Gautam SC, Chopp M. (2003a) Intravenous bone marrow stromal cell therapy reduces apoptosis and promotes endogenous cell proliferation after stroke in female rat. J Neurosci Res 73:778-786

Chen J, Li Y, Wang L, Lu M, Zhang X, Chopp M. (2001a) Therapeutic benefit of intracerebral transplantation of bone marrow stromal cells after cerebral ischemia in rats. J Neurol Sci 189:49-57

Chen J, Li Y, Wang L, Zhang Z, Lu D, Lu M, Chopp M. (2001b) Therapeutic benefit of intravenous administration of bone marrow stromal cells after cerebral ischemia in rats. Stroke 32:1005-1011

Chen J, Sanberg PR, Li Y, Wang L, Lu M, Willing AE, Sanchez-Ramos J, Chopp M. (2001c) Intravenous administration of human umbilical cord blood reduces behavioral deficits after stroke in rats. Stroke 32:2682-2688

Chen J, Zhang ZG, Li Y, Wang L, Xu YX, Gautam SC, Lu M, Zhu Z, Chopp M. (2003b) Intravenous administration of human bone marrow stromal cells induces angiogenesis in the ischemic boundary zone after stroke in rats. Circ Res 92:692-699

Chiang YH, Lin SZ, Borlongan CV, Hoffer BJ, Morales M, Wang Y. (1999) Transplantation of fetal kidney tissue reduces cerebral infarction induced by middle cerebral artery ligation. J Cereb Blood Flow Metab 19:13291335

Chopp M, Li Y. (2002) Treatment of neural injury with marrow stromal cells. Lancet Neurol 1:92-100

Han BH, Holtzman DM. (2000) BDNF protects the neonatal brain from hypoxic-ischemic injury in vivo via the ERK pathway. J Neurosci 20:5775-5781

Kilic U, Kilic E, Jarve A, Guo Z, Spudich A, Bieber K, Barzena U, Bassetti CL, Marti HH, Hermann DM. (2006) Human vascular endothelial growth factor protects axotomized retinal ganglion cells in vivo by activating ERK-1/2 and Akt pathways. J Neurosci 26:1243912446
Kovacsovics-Bankowski M, Mauch K, Raber A, Streeter PR, Deans RJ, Maziarz RT, Van't Hof W. (2008) Preclinical safety testing supporting clinical use of allogeneic multipotent adult progenitor cells. Cytotherapy 10:730-742

Kovacsovics-Bankowski M, Streeter PR, Mauch KA, Frey MR, Raber A, van't Hof W, Deans R, Maziarz RT. (2009) Clinical scale expanded adult pluripotent stem cells prevent graft-versus-host disease. Cell Immunol 255:55-60

Li Y, Chen J, Wang L, Lu M, Chopp M. (2001) Treatment of stroke in rat with intracarotid administration of marrow stromal cells. Neurology 56:1666-1672

Li Y, Chopp M. (2009) Marrow stromal cell transplantation in stroke and traumatic brain injury. Neurosci Lett 456:120-123

Lloyd-Jones D, Adams R, Carnethon M, De Simone G, Ferguson TB, Flegal K, Ford E, Furie K, Go A, Greenlund K, Haase N, Hailpern S, Ho M, Howard V, Kissela B, Kittner S, Lackland D, Lisabeth L, Marelli A, McDermott M, Meigs J, Mozaffarian D, Nichol G, O'Donnell C, Roger V, Rosamond W, Sacco R, Sorlie $\mathrm{P}$, Stafford R, Steinberger J, Thom T, WasserthielSmoller S, Wong N, Wylie-Rosett J, Hong Y. (2009) Heart disease and stroke statistics--2009 update: a report from the American Heart Association Statistics Committee and Stroke Statistics Subcommittee. Circulation 119:e21-181

Ohtaki H, Ylostalo JH, Foraker JE, Robinson AP, Reger RL, Shioda S, Prockop DJ. (2008) Stem/progenitor cells from bone marrow decrease neuronal death in global ischemia by modulation of inflammatory/immune responses. Proc Natl Acad Sci U S A 105:14638-14643

Savitz SI. (2009) Introduction to cellular therapy: the next frontier for stroke therapeutics. Stroke 40:S141-142

Shen LH, Li Y, Chen J, Zacharek A, Gao Q, Kapke A, Lu M, Raginski K, Vanguri P, Smith A, Chopp M. (2007) Therapeutic benefit of bone marrow stromal cells administered 1 month after stroke. J Cereb Blood Flow Metab 27:6-13

Shyu WC, Lee YJ, Liu DD, Lin SZ, Li H. (2006) Homing genes, cell therapy and stroke. Front Biosci 11:899907

Stem Cell Therapies as an Emerging Paradigm in Stroke (STEPS): bridging basic and clinical science for cellular and neurogenic factor therapy in treating stroke. (2009). Stroke 40:510-515

Stroemer P, Patel S, Hope A, Oliveira C, Pollock K, Sinden J. (2009) The Neural Stem Cell Line CTX0E03 Promotes Behavioral Recovery and Endogenous Neurogenesis After Experimental Stroke in a DoseDependent Fashion. Neurorehabil Neural Repair

Ting AE, Mays RW, Frey MR, Hof WV, Medicetty S, Deans R. (2008) Therapeutic pathways of adult stem cell repair. Crit Rev Oncol Hematol 65:81-93

Wang Y, Hayashi T, Chang CF, Chiang YH, Tsao LI, Su TP, Borlongan C, Lin SZ. (2001) Methamphetamine poten- 
tiates ischemia/reperfusion insults after transient middle cerebral artery ligation. Stroke 32:775-782

Willing AE, Lixian J, Milliken M, Poulos S, Zigova T, Song S, Hart C, Sanchez-Ramos J, Sanberg PR. (2003) Intravenous versus intrastriatal cord blood administration in a rodent model of stroke. J Neurosci Res 73:296-307

Yasuhara T, Hara K, Maki M, Mays RW, Deans RJ, Hess DC, Carroll JE, Borlongan CV. (2008) Intravenous grafts recapitulate the neurorestoration afforded by intracerebrally delivered multipotent adult progenitor cells in neonatal hypoxic-ischemic rats. J Cereb Blood Flow Metab

Yasuhara T, Matsukawa N, Yu G, Xu L, Mays RW, Kovach $\mathrm{J}$, Deans R, Hess DC, Carroll JE, Borlongan CV. (2006a) Transplantation of cryopreserved human bone marrow-derived multipotent adult progenitor cells for neonatal hypoxic-ischemic injury: targeting the hippocampus. Rev Neurosci 17:215-225

Yasuhara T, Matsukawa N, Yu G, Xu L, Mays RW, Kovach J, Deans RJ, Hess DC, Carroll JE, Borlongan CV. (2006b) Behavioral and histological characterization of intrahippocampal grafts of human bone marrowderived multipotent progenitor cells in neonatal rats with hypoxic-ischemic injury. Cell Transplant 15:231238

Zhao LR, Duan WM, Reyes M, Keene CD, Verfaillie CM, Low WC. (2002) Human bone marrow stem cells exhibit neural phenotypes and ameliorate neurological deficits after grafting into the ischemic brain of rats. Exp Neurol 174:11-20 